\title{
Evaluation of Lynch syndrome modifier genes in 748 MMR mutation carriers
}

\author{
Solene Houlle ${ }^{1,2}$, Françoise Charbonnier ${ }^{1,2}$, Estelle Houivet ${ }^{3}$, Julie Tinat ${ }^{1,2}$, Marie-Pierre Buisine ${ }^{4}$, \\ Olivier Caron $^{5}$, Jacques Benichou ${ }^{3}$, Stéphanie Baert-Desurmont ${ }^{1,2}$ and Thierry Frebourg ${ }^{\star, 1,2}$
}

\begin{abstract}
Several studies have reported that, in Lynch syndrome resulting from mutations of the mismatch repair (MMR) genes, a CA repeat $\leq 17$ within the IGF1 promoter, SNPs within the xenobiotic metabolizing enzyme gene CYP1A1 and SNPs on 8q23.3 and 11q23.1 modify colorectal cancer (CRC) risk in MMR mutation carriers. We analysed the impact of these polymorphisms on CRC risk in 748 French MMR mutation carriers derived from 359 families. We also analysed the effect of the Novel 1 SNP (18q21), which has recently been shown to increase CRC risk in the general population. We observed a significant difference in the CRC-free survival time between males and females, between MSH2 and MSH6 mutation carriers and between $\mathrm{MLH} 1$ and MSH6, indicating that this series is representative of Lynch syndrome. In contrast, the univariate log-rank test, as well as multivariate Cox model analysis controlling for familial aggregation and mutated MMR gene, year of birth and gender showed that the polymorphic alleles tested were not associated with a significant CRC risk increase, neither on the entire sample nor among males and females. This discrepancy with previous reports might be explained both by the genetic heterogeneity between the different populations analysed and the allelic heterogeneity of the MMR mutations. We conclude that genotyping of these polymorphisms is not useful to evaluate CRC risk in MMR mutation carriers and to optimize their clinical follow-up.
\end{abstract} European Journal of Human Genetics (2011) 19, 887-892; doi:10.1038/ejhg.2011.44; published online 16 March 2011

Keywords: Lynch syndrome; MMR genes; cancer risk; modifier factors

\section{INTRODUCTION}

Lynch syndrome or hereditary non-polyposis colorectal cancer, the most common form of inherited colorectal cancer (CRC), results from germline mutations within the genes of the mismatch repair (MMR) system, MSH2, MLH1, MSH6 and PMS2 (for a review, see Lynch et al ${ }^{1}$ ). In MMR mutation carriers, the main tumour risks are colorectal and endometrial cancers. The cumulative risk at 70 years of CRC has been estimated to be $47-78 \%$ in males ${ }^{2-4}$ and $30-57 \%$ in females, ${ }^{2-4}$ and the risk of endometrial cancer to be $25-61 \%{ }^{2,3}$ Like in other Mendelian forms of cancer characterized by an incomplete penetrance, one of the main challenges is to identify modifier genetic factors that can modulate the mutation penetrance. Characterization of validated modifier genetic factors should have important clinical consequences in the future since it should then be possible to adapt the follow-up of MMR gene mutation carriers, in terms of nature and timing of investigations, according to modifier alleles.

The modifier genes that have been reported so far in Lynch syndrome have been characterized according to two strategies: several studies have been focused on genes whose implication in CRC development had previously been suggested. One of the first modifier genetic factors identified in Lynch syndrome corresponds to a CA repeat polymorphism present within the IGF1 promoter, $1 \mathrm{~kb}$ upstream from the transcriptional initiation site. ${ }^{5,6}$ In a study performed in 121 MMR mutation carriers from 59 families, mainly of Caucasian origin, Zecevic et $a l^{5}$ reported that a CA repeat $\leq 17$ was significantly associated with a higher CRC risk and an earlier age of tumour onset. This result was confirmed by an independent study performed in 443 Australian and Polish MMR mutation carriers originated from 269 distinct families. ${ }^{6}$ Another class of modifier genes reported in Lynch syndrome corresponds to genes encoding xenobiotic metabolizing enzymes involved in environmental carcinogen metabolism. In 129 subjects of South African origin and harbouring the same MLH1 missense mutation, males harbouring the null genotype for the GSTT1 and GSTM1 genes developed cancer earlier than the males harbouring the other genotypes. ${ }^{7}$ This effect of the GSTT1 and GSTM1 null genotypes was not confirmed in a second study including 257 MMR mutation carriers from 130 families. ${ }^{8}$ Nevertheless, the authors reported that subjects heterozygous for the CYP1A1 rs1048943 SNP (c.1384A>G; p.Ile462Val) developed CRC earlier than individuals with the homozygous wild-type genotype and that subjects heterozygous for this polymorphism and an additional SNP rs4646903 (Msp1; g.6235T >C) had an increased CRC risk. ${ }^{8}$

The second strategy, which has recently allowed the detection of modifier genes in Lynch patients, originated from the numerous genome-wide association studies reporting SNPs associated with $\mathrm{CRC}$ risk in the general population. Starting from the hypothesis that SNPs acting as risk factors for CRC in the general population might act as risk modifiers in patients harbouring a highly penetrant mutation, Wijnen et al ${ }^{9}$ recently reported a significant association of CRC risk with rs16892766 (8q23.3) and rs3802842 (11q23.1) in 675 Dutch MMR mutation carriers from 127 families.

\footnotetext{
${ }^{1}$ Inserm U614, Faculty of Medicine, Institute for Biomedical Research and Innovation, University of Rouen, Rouen, France; ${ }^{2}$ Department of Genetics, University Hospital, Rouen, France; ${ }^{3}$ Department of Biostatistics and Inserm U657, University Hospital, Rouen, France; ${ }^{4}$ Laboratory of Molecular Biology, University Hospital, Lille, France; ${ }^{5}$ Department of Medicine, Institut Gustave Roussy, Villejuif, France

${ }^{*}$ Correspondence: Professor T Frebourg, Inserm U614, Faculty of Medicine, Institute for Biomedical Research and Innovation, University of Rouen, 22 Boulevard Gambetta, Rouen, 76183, France. Tel: +33 2328881 82; Fax: +33 2328880 80; E-mail: Frebourg@chu-rouen.fr
}

Received 20 September 2010; revised 26 January 2011; accepted 27 January 2011; published online 16 March 2011 
In this study, we investigated the impact of these different genetic factors on CRC risk in a large series of French MMR mutation carriers.

\section{PATIENTS AND METHODS}

\section{Patients}

The study included 748 unselected patients (Table 1) derived from 359 families with Lynch syndrome and recruited from Rouen $(n=494)$ and Lille $(n=254)$ university hospitals in France. All these individuals were confirmed carriers of a deleterious mutation of MSH2, MLH1 or MSH6 and were Caucasian. Among these 748 mutation carriers, 329 (44\%) had been diagnosed with CRC prior to inclusion in the study, with a mean age of CRC onset of 43 years (range: 18-82 years). Additionally, 51 mutation carriers $(6.8 \%)$ had been diagnosed with another tumour belonging to the Lynch syndrome spectrum (endometrial carcinoma, tumour of the urinary tract, ovarian carcinoma, cancer of the stomach and of the small intestine) and 368 (49.2\%) had developed no tumour at the time of inclusion.

\section{Genotyping}

The IGF1 CA repeat was PCR-amplified using dye-labelled primers and the length of the PCR products was determined after migration on an Applied Biosystems model 3130 Genetic Analyser (PE Applied Biosystems, Foster City, CA, USA) and analysed using the GeneMapper Analysis Software version 4.0 (Applied Biosystems). To calibrate the results, we sequenced, after cloning into a plasmid, the most frequent allele $(n=19)$ and used the corresponding genomic DNA as a reference DNA. The rs16892766 (8q23.3), rs3802842 (11q23.1) SNPs and the rs1048943 (c.1384A>G; p.Ile462Val) and rs4646903 SNPs, both located within the CYP1A1 gene (15q24.1), were genotyped using SNaPshot multiplex assays based on primer extension with dye-labelled dideoxynucleotides (ABI PRISM SNaPshot Multiplex kit, Applied Biosystems). We also analysed, by SNaPshot multiplex analysis, the Novel 1 SNP located on 18q21, which had been shown to be associated with CRC risk in the general population, by altering $S M A D 7$ expression. ${ }^{10}$ Labelled products were separated

Table 1 Clinical characteristics of MMR mutation carriers

\begin{tabular}{|c|c|c|c|}
\hline & $\begin{array}{c}\text { Number of } \\
\text { subjects (\%) } \\
(\mathrm{n}=748)\end{array}$ & $\begin{array}{c}\text { Number of } \\
\text { incident CRC } \\
\text { cases }(\mathrm{n}=329)\end{array}$ & $\begin{array}{c}\text { Log-rank test, } \\
\text { P-value }\end{array}$ \\
\hline \multicolumn{4}{|l|}{ Gender } \\
\hline Male & $349(46.7)$ & 177 & $<0.0001$ \\
\hline Female & 399 (53.3) & 152 & \\
\hline \multicolumn{4}{|l|}{ Index case } \\
\hline Yes & $290(38.8)$ & 259 & \\
\hline No & $458(61.2)$ & 70 & \\
\hline \multicolumn{4}{|c|}{ MMR gene mutated } \\
\hline MSH2 & $414(55.4)$ & 178 & 0.0495 (vs MSH6) \\
\hline$M L H 1$ & $267(35.7)$ & 115 & 0.0069 (vs MSH6) \\
\hline MSHG & $67(8.9)$ & 36 & \\
\hline \multicolumn{4}{|l|}{ Year of birth } \\
\hline$<1940$ & $57(7.6)$ & $39(11.8)$ & \\
\hline 1940-1949 & $106(14.2)$ & $73(22.2)$ & \\
\hline 1950-1959 & $188(25.1)$ & $119(36.2)$ & \\
\hline 1960-1969 & $165(22.1)$ & $66(20.1)$ & \\
\hline 1970-1979 & $129(17.2)$ & $26(7.9)$ & \\
\hline$\geq 1980$ & $103(13.8)$ & $6(1.8)$ & \\
\hline Age & & Mean, $\min / \max$ & \\
\hline With CRC & $329(44)$ & $43,18 / 82$ & \\
\hline Without CRC & $419(56)$ & $38,18 / 78$ & \\
\hline
\end{tabular}

using a 25-min run on an ABI Prism 3100 DNA sequencer and data were analysed using the GeneMapper Analysis Software version 4.0 (Applied Biosystems). Primer sequences used for genotyping and conditions of the $\mathrm{SNaPshot}$ multiplex assays are available on request.

\section{Statistical analyses}

The genotype frequencies were tested for Hardy-Weinberg equilibrium. In all following analyses of time to CRC, first CRC onset was the event of interest, age (in years) was used at the time scale and retrospective follow-up started at birth for all subjects in the study and ended at either first CRC onset for incident CRC cases or age at last follow-up otherwise. These analyses were performed for all subjects and separately for males and females and were repeated for CRC cases only. The association of sex or mutated MMR gene with CRC risk was assessed using the log-rank test. The association of each polymorphism (ie, five SNPs and IGF1 CA repeat length) with CRC risk was assessed using the log-rank test and then the Cox proportional hazard model with stratification on gender (if applicable), mutated MMR gene (MSH2, MLH1, MSH6) and date of birth (<1940, 1940-1949, 1950-1959, 1960-1969, 1970-1979 and $\geq 1980$ ) and with control for familial clustering. In the Cox model analyses of each of the five SNPs, an overall comparison of the three genotypes was performed and a hazard ratio (HR) and corresponding 95\% confidence interval were estimated

Table 2 Allelic frequency of genetic variants and assessment of the associated colorectal risk by the log-rank test

\begin{tabular}{llll}
\hline Genetic variant & Allelic frequency & $\begin{array}{c}\text { Log-rank test } \mathrm{P} \text {-value } \\
\text { (n=748) }\end{array}$ & $\begin{array}{c}\text { CRC patients } \\
(\mathrm{n}=329)\end{array}$ \\
\hline rs16892766 (8q23.3) & & & \\
MAF & 0.07 (C allele) & & \\
$\quad$ Total sample & & 0.2188 & 0.0946 \\
$\quad$ Males & & 0.3146 & 0.5209 \\
Females & & 0.7409 & 0.0585
\end{tabular}

rs3802842 (11q23.1)

MAF

0.28 ( $C$ allele)

Total sample

Males

Females

0.6206

0.8345

0.2316

0.7557

0.7805

0.4081

Novel 1 (18q21)

MAF

Total sample

0.47 ( $C$ allele)

Males

Females

0.9698

0.4676

0.3560

0.8847

0.3483

0.2349

rs1048943

MAF

0.03 ( $G$ allele)

Total sample

Males

0.7632

0.8957

0.3442

Females

0.9003

0.5035

0.6082

rS4646903 (CYP1A)

MAF

0.12 ( $C$ allele)

Total sample

Males

0.9489

0.6490

Females

0.3701

0.7074

$0.6772 \quad 0.5724$

IGF1 CA repeat

MAF

Total sample

Males

0.5763

0.6002

Females

0.2918

0.1562

0.9457 0.4249 
for homozygous and heterozygous subjects, relative to subjects homozygous for the wild-type allele. Moreover, alternative Cox model analyses of each of the five SNPs considered the number of alleles with the $\operatorname{SNP}(0,1,2)$ and fitted a trend producing an alternative test of association with CRC risk and an estimate of the CRC HR per mutated allele. Statistical analyses were performed using the SAS software version 9.1 (SAS Institute, Cary, NC, USA).

\section{RESULTS}

Among the 748 MMR mutation carriers included in this study, 329 carriers (44\%) developed CRC. As shown in Table 1, a significant difference was observed in the age at first CRC occurrence between males and females, with males developing CRC earlier, between $\mathrm{MSH} 2$ and MSH6 mutation carriers, between MLH1 and MSH6 mutation carriers, with $\mathrm{MSH} 2$ and $\mathrm{MLH} 1$ carriers developing CRC earlier, but not between $\mathrm{MSH} 2$ and $\mathrm{MLH1}$ mutation carriers (log-rank test, $P=0.26$ ). The five SNPs analysed, located on $8 \mathrm{q} 23.3,11 \mathrm{q} 23.1,18 \mathrm{q} 21$ and within CYP1A1, were in Hardy-Weinberg equilibrium. The distribution of the IGF1 CA repeat alleles was the following: 12 repeats $(n=8), 13-15$ repeats $(n=0), 16$ repeats $(n=2), 17$ repeats $(n=28)$, 18 repeats $(n=85), 19$ repeats $(n=976), 20$ repeats $(n=262), 21$ repeats $(n=114)$ and 22 repeats $(n=21)$ and alleles were dichotomized in $\leq 17$ and $\geq 18$ repeats for the statistical analyses, like in the previous studies. ${ }^{5,6}$

In univariate analysis, age at first CRC occurrence did not vary significantly for the five SNPs considered or according to number of IGF1 CA repeats, whether for the whole sample or separately for males and females (Table 2). As indicated in Table 3, the multivariate Cox analysis performed in the total sample of mutation carriers revealed that, for rs16892766 (8q23.3), the homozygosity for the minor $C$ allele present only in three mutation carriers was significantly associated with a decreased risk relative to subjects with $A A$ alleles $(H R=0.267$, $P=0.0271)$. For the CYPA1 SNPs, we observed a significant difference

Table 3 Evaluation in 748 MMR mutation carriers of the CRC risk associated with 8q23.3, 11q23.1, 18q21, CYP1A1 and IGF1 variants

\begin{tabular}{|c|c|c|c|c|c|c|c|c|c|c|c|c|c|c|c|c|}
\hline \multirow[b]{2}{*}{ Locus } & \multirow[b]{2}{*}{ Genotype } & \multicolumn{5}{|c|}{ Total sample $(\mathrm{N}=748)$} & \multicolumn{5}{|c|}{ Males $(\mathrm{N}=349)$} & \multicolumn{5}{|c|}{ Females $(N=399)$} \\
\hline & & $\mathrm{N}$ & $\begin{array}{l}\text { CRC } \\
\text { cases }\end{array}$ & $\begin{array}{c}\text { Hazard } \\
\text { ratio }\end{array}$ & $\begin{array}{c}95 \% \\
\mathrm{Cl}\end{array}$ & $P^{b}$ & $\mathrm{~N}$ & $\begin{array}{l}\text { CRC } \\
\text { Cases }\end{array}$ & $H R$ & $\begin{array}{c}95 \% \\
\mathrm{Cl}\end{array}$ & $\mathrm{P}^{\mathrm{b}}$ & $\mathrm{N}$ & $\begin{array}{c}\text { CRC } \\
\text { cases }\end{array}$ & $H R$ & $\begin{array}{c}95 \% \\
\mathrm{Cl}\end{array}$ & $\mathrm{P}^{\mathrm{b}}$ \\
\hline \multirow[t]{6}{*}{$8 q 23.3$} & rs16892766 & & & & & & & & & & & & & & & \\
\hline & $A A$ & 641 & 277 & 1 & & & 290 & 142 & 1 & & & 351 & 135 & 1 & & \\
\hline & $A C$ & 104 & 50 & 1.184 & $0.866-1.617$ & 0.2892 & 57 & 34 & 1.266 & $0.836-1.916$ & 0.2656 & 47 & 16 & 1.067 & $0.677-1.680$ & 0.7802 \\
\hline & $C C$ & 3 & 2 & 0.267 & $0.083-0.861$ & 0.0271 & 2 & 1 & 0.460 & $0.301-0.701$ & 0.0003 & 1 & 1 & I & l & $<0.0001$ \\
\hline & & & & & & 0.0462 & & & & & 0.0002 & & & & & $<0.0001$ \\
\hline & Per allele & & & 1.032 & $0.778-1.368$ & 0.8283 & & & 1.117 & $0.770-1.621$ & 0.5602 & & & 0.899 & $0.575-1.405$ & 0.6397 \\
\hline \multirow[t]{6}{*}{ 11q23.1 } & $r s 3802842$ & & & & & & & & & & & & & & & \\
\hline & $A A$ & 380 & 167 & 1 & & & 178 & 90 & 1 & & & 202 & 77 & 1 & & \\
\hline & $A C$ & 321 & 139 & 0.923 & $0.725-1.176$ & 0.5165 & 141 & 71 & 0.844 & $0.611-1.167$ & 0.3047 & 180 & 68 & 1.019 & $0.723-1.438$ & 0.9125 \\
\hline & $C C$ & 47 & 23 & 1.107 & $0.684-1.791$ & 0.6793 & 30 & 16 & 1.043 & $0.599-1.815$ & 0.8814 & 17 & 7 & 1.222 & $0.543-2.750$ & 0.6284 \\
\hline & & & & & & 0.6491 & & & & & 0.5079 & & & & & 0.8891 \\
\hline & Per allele & & & 0.988 & $0.806-1.210$ & 0.9058 & & & 0.947 & $0.735-1.220$ & 0.6740 & & & 1.052 & $0.778-1.422$ & 0.7428 \\
\hline \multirow[t]{6}{*}{$18 q 21$} & Novel 1 & & & & & & & & & & & & & & & \\
\hline & $C C$ & 156 & 72 & 1 & & & 84 & 45 & 1 & & & 72 & 27 & 1 & & \\
\hline & $C G$ & 395 & 165 & 0.956 & $0.704-1.296$ & 0.7702 & 185 & 87 & 1.021 & $0.675-1.546$ & 0.9215 & 210 & 78 & 0.865 & $0.546-1.370$ & 0.5359 \\
\hline & $G G$ & 197 & 92 & 1.006 & $0.727-1.390$ & 0.9729 & 80 & 45 & 1.088 & $0.706-1.677$ & 0.7007 & 117 & 47 & 0.903 & $0.564-1.447$ & 0.6714 \\
\hline & & & & & & 0.9001 & & & & & 0.9087 & & & & & 0.8249 \\
\hline & Per allele & & & 1.007 & $0.859-1.181$ & 0.9326 & & & 1.044 & $0.841-1.295$ & 0.6962 & & & 0.964 & $0.765-1.215$ & 0.7570 \\
\hline \multirow[t]{6}{*}{ CYP1A1 } & rs1048943 & & & & & & & & & & & & & & & \\
\hline & $A A$ & 708 & 313 & 1 & & & 331 & 168 & 1 & & & 377 & 145 & 1 & & \\
\hline & $A G$ & 40 & 16 & 1.026 & $0.601-1.752$ & 0.9249 & 18 & 9 & 0.930 & $0.444-1.948$ & 0.8480 & 22 & 7 & 1.168 & $0.583-2.338$ & 0.6619 \\
\hline & $G G$ & 0 & 0 & 1 & l & 1 & 0 & 0 & I & l & 1 & 0 & 0 & I & l & I \\
\hline & & & & & & 0.9249 & & & & & 0.8480 & & & & & 0.6619 \\
\hline & Per allele & & & 1.026 & $0.601-1.752$ & 0.9249 & & & 0.930 & $0.444-1.948$ & 0.8480 & & & & & \\
\hline \multirow[t]{6}{*}{ CYP1A1 } & $r s 4646903$ & & & & & & & & & & & & & & & \\
\hline & $T T$ & 598 & 265 & 1 & & & 279 & 143 & 1 & & & 319 & 122 & 1 & & \\
\hline & $T C$ & 140 & 61 & 1.031 & $0.769-1.382$ & 0.8400 & 66 & 32 & 0.909 & $0.601-1.375$ & 0.6524 & 74 & 29 & 1.198 & $0.789-1.818$ & 0.3956 \\
\hline & $C C$ & 10 & 3 & 1.163 & $0.387-3.495$ & 0.7882 & 4 & 2 & 3.496 & $1.517-8.057$ & 0.0033 & 6 & 1 & 0.507 & $0.071-3.640$ & 0.4997 \\
\hline & & & & & & 0.9441 & & & & & 0.0090 & & & & & 0.5434 \\
\hline & Per allele & & & 1.039 & $0.801-1.347$ & 0.7730 & & & 1.001 & $0.680-1.474$ & 0.9948 & & & 1.079 & $0.746-1.561$ & 0.6874 \\
\hline \multirow[t]{3}{*}{ IGFI } & $C A$ repeat & & & & & & & & & & & & & & & \\
\hline & $\geq 18 ; \geq 18$ & 710 & & 1 & & & 329 & & 1 & & & 381 & & 1 & & \\
\hline & $\geq 18 ; \leq 17$ & 38 & & 0.731 & $0.447-1.196$ & 0.2127 & 20 & & 0.662 & $0.372-1.179$ & 0.1611 & 18 & & 0.979 & $0.450-2.132$ & 0.9579 \\
\hline
\end{tabular}


only in males for one of the SNPs analysed (rs4646903): the CC genotype, detected in four male mutation carriers, was associated with an increased risk relative to subjects with $T T$ alleles $(\mathrm{HR}=3.496$, $P=0.0033$ ). Considering that penetrance of MSH6 mutation was found in this study to be lower than that of $M S H 2$ and $M L H 1$ mutations, we restricted the log-rank and Cox analyses to MSH2 and MLH1 carriers and this restriction did not modify the results (data not shown).

As colonoscopy had not been performed in all the unaffected mutation carriers at the time of this study, we then focused the statistical analyses on the 329 MMR mutation carriers with CRC, in order to avoid a bias in the phenotypic evaluation. As indicated in Table 4, the unrestricted multivariate Cox analysis showed that the only remaining significant association was a decreased risk associated with the 8q23.3 CC genotype, but it should be emphasized that, because this genotype was only carried by two CRC cases, no HR estimate could be produced.

\section{DISCUSSION}

Like in other cases of Mendelian predisposition to cancer with an incomplete penetrance, the characterization of modifier loci for CRC in Lynch syndrome might allow identification of a subset of mutation carriers who could benefit from a reinforced tumour detection program, such as annual chromocolonoscopy from 20 years of age. This prospect prompted us to evaluate in a large series of $748 \mathrm{MMR}$ carriers the risk conferred by the allelic variants that have recently been reported as risk modifiers for CRC in MMR mutation carriers. Within this series, $44 \%$ of the MMR mutation carriers had developed CRC at the time of the analysis; the median age of CRC onset was 43 years among them and we observed a significant difference of CRC penetrance between males and females and between $\mathrm{MSH} 2$ and MLH1 mutation versus MSH6 mutation carriers. These results are in agreement with the published studies, which have estimated that the cumulative CRC risk at 70 years is higher in males than in

Table 4 Evaluation in 329 MMR mutation carriers with CRC of the risk associated with 8q23.3, 11q23.1, 18q21, CYP1A1 and IGF1 variants

\begin{tabular}{|c|c|c|c|c|c|c|c|c|c|c|c|c|c|}
\hline \multirow[b]{3}{*}{ Locus } & \multirow[b]{3}{*}{ Genotype } & \multicolumn{4}{|c|}{ Total sample $(\mathrm{N}=329)$} & \multicolumn{4}{|c|}{ Males $(\mathrm{N}=177)$} & \multicolumn{4}{|c|}{ Females $(\mathrm{N}=152)$} \\
\hline & & & Hazard & $95 \%$ & & & & $95 \%$ & & & & $95 \%$ & \\
\hline & & $\mathrm{N}$ & ratio ${ }^{a}$ & $\mathrm{Cl}$ & $\mathrm{P}^{\mathrm{b}}$ & $\mathrm{N}$ & $H R$ & $\mathrm{Cl}$ & $\mathrm{P}^{\mathrm{b}}$ & $\mathrm{N}$ & $H R$ & $\mathrm{Cl}$ & $\mathrm{P}^{\mathrm{b}}$ \\
\hline \multirow[t]{6}{*}{$8 q 23.3$} & rs16892766 & & & & & & & & & & & & \\
\hline & $A A$ & 277 & 1 & & & 142 & 1 & & & 135 & 1 & & \\
\hline & $A C$ & 50 & 0.793 & $0.559-1.124$ & 0.1920 & 34 & 0.768 & $0.478-1.234$ & 0.2749 & 16 & 0.833 & 0.5041 .376 & 0.4759 \\
\hline & $C C$ & 2 & 1 & l & $<0.0001$ & 1 & I & 1 & $<0.0001$ & 1 & 1 & 1 & $<0.0001$ \\
\hline & & & & & $<0.0001$ & & & & $<0.0001$ & & & & $<0.0001$ \\
\hline & Per allele & & 0.666 & $0.481-0.922$ & 0.0144 & & 0.647 & $0.414-1.009$ & 0.0549 & & 0.698 & $0.443-0.102$ & 0.1227 \\
\hline \multirow[t]{6}{*}{$11 \mathrm{q} 23.1$} & $r s 3802842$ & & & & & & & & & & & & \\
\hline & $A A$ & 167 & 1 & & & 90 & 1 & & & 77 & 1 & & \\
\hline & $A C$ & 139 & 0.941 & $0.738-1.199$ & 0.6216 & 71 & 0.840 & $0.593-1.191$ & 0.3274 & 68 & 1.067 & $0.751-1.515$ & 0.7180 \\
\hline & $C C$ & 23 & 1.234 & $0.694-2.195$ & 0.4736 & 16 & 1.034 & $0.548-1.950$ & 0.9182 & 7 & 1.815 & $0.664-4.963$ & 0.2457 \\
\hline & & & & & 0.6151 & & & & 0.5740 & & & & 0.5057 \\
\hline & Per allele & & 1.028 & $0.831-1.272$ & 0.9763 & & 0.947 & $0.723-1.239$ & 0.947 & & 1.170 & $0.854-1.604$ & 0.3289 \\
\hline \multirow[t]{6}{*}{$18 q 21$} & Novel 1 & & & & & & & & & & & & \\
\hline & $C C$ & 72 & 1 & & & 45 & 1 & & & 27 & 1 & & \\
\hline & $C G$ & 165 & 0.828 & $0.588-1.166$ & 0.2804 & 87 & 1.081 & $0.701-1.667$ & 0.7249 & 78 & 0.571 & $0.315-1.035$ & 0.0647 \\
\hline & $G G$ & 95 & 0.741 & $0.516-1.062$ & 0.1029 & 45 & 0.867 & $0.554-1.358$ & 0.5326 & 47 & 0.570 & $0.305-1.068$ & 0.0793 \\
\hline & & & & & 0.2637 & & & & 0.5573 & & & & 0.1637 \\
\hline & Per allele & & 0.865 & $0.726-1.031$ & 0.1056 & & 0.919 & $0.735-1.149$ & 0.4607 & & 0.796 & $0.595-1.065$ & 0.1238 \\
\hline \multirow[t]{6}{*}{ CYP1A1 } & rs1048943 & & & & & & & & & & & & \\
\hline & $A A$ & 313 & 1 & & & 168 & 1 & & & 145 & 1 & & \\
\hline & $A G$ & 16 & 1.107 & $0.609-2.011$ & 0.7387 & 9 & 1.105 & $0.502-2.432$ & 0.8045 & 7 & 1.110 & $0.447-2.754$ & 0.8224 \\
\hline & $G G$ & 0 & I & l & 1 & 0 & I & 1 & I & 0 & I & l & 1 \\
\hline & & & & & 0.7387 & & & & 0.8045 & & & & 0.8224 \\
\hline & Per allele & & 1.107 & $0.609-2.011$ & 0.7387 & & 1.105 & $0.502-2.432$ & 0.8045 & & 1.110 & $0.447-2.754$ & 0.8224 \\
\hline \multirow[t]{6}{*}{ CYP1A1 } & rs4646903 & & & & & & & & & & & & \\
\hline & $T T$ & 265 & 1 & & & 143 & 1 & & & 122 & 1 & & \\
\hline & $T C$ & 61 & 0.988 & $0.725-1.348$ & 0.9412 & 32 & 1.070 & $0.696-1.647$ & 0.7568 & 29 & 0.902 & $0.572-1.421$ & 0.6558 \\
\hline & $C C$ & 3 & 1.741 & $0.925-3.278$ & 0.0856 & 2 & 2.276 & $0.890-5.822$ & 0.0861 & 1 & 1.241 & $0.759-2.029$ & 0.3895 \\
\hline & & & & & 0.2094 & & & & 0.2291 & & & & 0.5036 \\
\hline & Per allele & & 1.038 & $0.786-1.372$ & 0.7919 & & 1.139 & $0.773-1.678$ & 0.5117 & & 0.930 & $0.618-1.399$ & 0.7277 \\
\hline \multirow[t]{3}{*}{ IGF1 } & $C A$ repeat & & & & & & & & & & & & \\
\hline & $\geq 18 ; \geq 18$ & 314 & 1 & & & 166 & 1 & & & 148 & 1 & & \\
\hline & $\geq 18 ; \leq 17$ & 15 & 0.710 & $0.397-1.272$ & 0.2502 & 11 & 0.562 & $0.297-1.063$ & 0.0765 & 4 & 1.709 & $0.470-6.212$ & 0.4160 \\
\hline
\end{tabular}

aHazard ratio relative to subjects not carrying the SNP on either allele for each of the five SNPs considered or to subjects with CA repeat length greater than or equal to 18 on both alleles for the IGF1 promoter and obtained from Cox proportional hazard regression stratified on gender (if applicable), mutated MMR gene (MSH2, MLH1, MSH6) and year of birth (<1940, 1940-1949, 1950-1959, 1960-1969, 1970-1979 and $\geq 1980$ ), and with control for familial aggregation. The per-allele value refers to Cox regression with a trend fitted for number of alleles carrying the $\operatorname{SNP}(0,1,2)$

bFor each of the five SNP variants and each sample (total sample, males, females), the first two $P$-values refer to separate comparisons of heterozygous and homozygous subjects for the SNPs considered, with subject not presenting the SNP on either allele; the third $P$-value refers to the overall comparison of heterozygous, homozygous and SNP-free subjects (heterogeneity test); the final $P$-value refers to the assessment of the trend for the number of alleles carrying the SNP $(0,1,2)$. For IGFI CA repeat length, the $P$-value refers to the comparison of subjects with CA repeat length less than or equal to 17 on one allele to subjects with CA repeat length greater than or equal to 18 on both alleles. All tests are Wald tests. 
females $^{2-4}$ and is lower in MSH6 mutation carriers as compared with $M S H 2$ or $M L H 1$ mutation carriers. ${ }^{2,3,11,12}$ These findings are in favour of the representativeness of our series with respect to Lynch syndrome.

The distribution of IGF1 CA repeat length was similar to that reported by Zecevic et al ${ }^{4}$ in 121 MMR mutation carriers, the frequency of the major allele (19 repeats) being 65 and $58 \%$ in the two studies, respectively, although we found a lower proportion of alleles with length $\leq 17$ (2.5 versus $5.1 \%)$. In the study published by Zecevic et $a l^{5}$ on $121 \mathrm{MMR}$ mutation carriers, the presence of a CA repeat $\leq 17$, detected in 12 subjects, was found to be associated with a higher CRC risk (HR 2.36) and an earlier median age of CRC onset (44 versus 56.5 years), but our study, performed on a larger series, did not allow us to reproduce these results.

For the rs1048943 (c.1384A> G; p.Ile462Val) and rs4646903 SNPs, located within the CYP1A1 gene, we found respective allelic frequencies for the minor allele of 0.03 and 0.12 , which correspond to the HapMap values estimated in the European population. Pande et al ${ }^{8}$ had reported a higher MAF (0.074) for rs1048943 in 257 MMR mutation carriers, but it should be underlined that these carriers were from different ethnicities and included Hispanic and Asian subjects, in whom the allelic frequency of the $G$ allele is known to be higher. In this series of 257 mutation carriers, CRC free survival statistically differed according to rs1048943 genotype, with a 1.78-fold increase of CRC hazard associated with the $A G$ genotype, a result that we did not reproduce in our series of 748 MMR mutation carriers from Caucasian origin. For the second CYPA1 SNP (rs4646903), Pande et $a l^{8}$ had reported that the TC genotype was associated with an increased hazard for earlier CRC and we detected no significant difference in our study for this genotype (Table 3). In contrast, we found that the $C C$ genotype was associated in males with a 3.5-fold increased HR, but this observation should be interpreted with utmost caution, considering the limited number of male mutation carriers presenting this genotype $(n=4)$. For rs16892766 (8q23.3) and rs3802842 (11q23.1) SNPs, we observed respective allele frequencies of 7 and 28\%: these frequencies had been, respectively, estimated in the European population at $7 \%{ }^{13}$ and $29 \%,{ }^{14}$ and in 675 Dutch MMR mutation carriers at 10 and $24.9 \% .{ }^{9}$ We did not detect in our series a modification of CRC risk in mutation carriers harbouring the 11q23.1 rs3802842 CC genotype, as previously reported in females. Among the 675 Dutch mutation carriers, the 8q23.3 rs16892766 CC genotype, detected in 9 subjects, was found to be associated with a 2.16-fold CRC risk increase. ${ }^{9}$ In contrast, we found in our study (Table 3) that this genotype was significantly associated with a decreased CRC risk $(\mathrm{HR}=0.267)$, and this significant association was the only one remaining when we restricted the analysis to affected carriers, but the small number of subjects harbouring this genotype $(n=3)$ and presenting a CRC $(n=2)$ must be highlighted.

During the submission of this study, Talseth-Palmer et al ${ }^{15}$ reported that in $M L H 1$ carriers, but not in MSH2 carriers, the 11q23.1 CC and 8q23.3 AC genotypes were associated with an increased risk, but this significant association detected in 373 Australian mutation carriers was not found in 311 Polish mutation carriers analysed in the same study. We did not replicate either of these associations in the French sample. Indeed, the only association that we detected for these two SNPs in the 267 French $M L H 1$ mutation carriers was a decrease of CRC risk with the 8q23.3 CC genotype $(P<0.0001)$, but as this genotype was carried by only one CRC case, it was not possible to estimate the corresponding HR and due caution should be applied to interpret this finding.
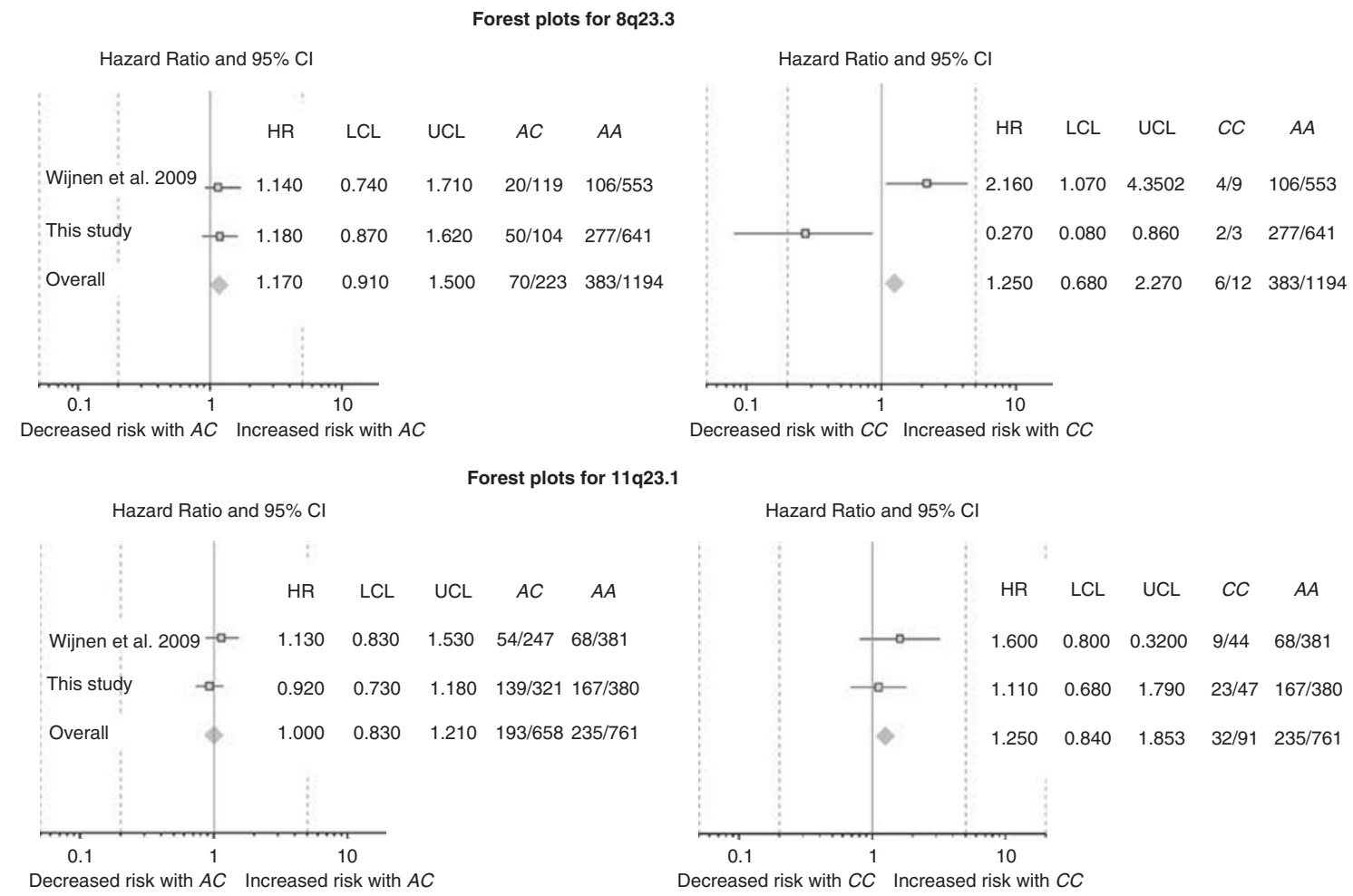

Figure 1 Forest plots of the meta-analysis of the $C R C$ risk associated with 8q23.3 and 11q23.1 variants in $1423 \mathrm{MMR}$ mutation carriers. Cl, confidence interval; HR, hazard rate; LCL, lower 95\% confidence limit; UCL, upper $95 \%$ confidence limit. For each panel, the last two columns display the number of subjects with colorectal cancer and the indicated genotype, followed by the overall number of subjects with this genotype. 
To increase the power of statistical analysis of the $8 \mathrm{q} 23.3$ and 11q23.1 SNPs' impact on CRC risk in $M M R$ mutation carriers, we performed a meta-analysis based on the study published by Wijnen et $a l^{9}$ and our results, corresponding to 1423 MMR mutation carriers (Figure 1). In univariate analysis, age at first CRC occurrence varied significantly in male carriers according to the 8q23.3 genotype ( $A A, A C$ or $C C ; P=0.0265$ using Fisher's standard method to combine $P$-values from two log-rank tests), with increased CRC risk associated with the $A C$ genotype and decreased risk associated with the $C C$ genotype. In multivariate Cox analysis, as shown on the Forest plot for the total sample (Figure 1), CRC risk did not vary significantly according to the $8 \mathrm{q} 23.3$ genotype. In males, the multivariate Cox analysis showed that the 8q23.3 CC genotype was associated with a decrease in CRC risk (combined fixed-effect weighted sum $\mathrm{HR}=0.61$ relative to $A A, P=0.0135$ ). It remains, however, that there were very few males and even fewer male CRC cases carrying the $C C$ genotype in both studies and thus the results of this meta-analysis should be interpreted with caution. Univariate and multivariate Cox analyses did not reveal any significant association between CRC risk and the 11q23.1 genotype (Figure 1).

In conclusion, the evaluation in a series of $748 \mathrm{MMR}$ mutation carriers representative of Lynch syndrome of the IGF1 CA repeat, CYP1A1, 8q23.3 and 11q23.1 SNPs, previously reported as modifier factors for CRC risk in MMR mutation carriers, did not allow us to reproduce the previously published results. The only significant association that we detected both in the whole sample and in the unbiased affected mutation carrier sample was between the 8q23.3 CC genotype and a decreased CRC risk in males, whereas previously published studies had reported an increased CRC risk associated either with this genotype ${ }^{9}$ or with the heterozygous $A C$ genotype. ${ }^{15}$ This discrepancy might be explained by the genetic heterogeneity between the different analysed populations. Considering that, in MMR mutation carriers, cancer development requires somatic mutation of several target genes containing repeated sequences, it is likely that numerous polymorphisms affect either the DNA repair or target genes and/or that environmental factors having a role in colorectal carcinogenesis will modify the penetrance of MMR mutations. It is also possible that a key factor modulating the penetrance is the allelic heterogeneity of the MMR mutations. Finally, another explanation of this discrepancy between our results and previous studies is that the previously reported associations were spurious associations due to too small samples. Therefore, we conclude that, from the medical point of view, genotyping of these polymorphisms is not useful to evaluate CRC risk in MMR mutation carriers and to optimize their clinical follow-up.

\section{CONFLICT OF INTEREST}

The authors declare no conflict of interest.

\section{ACKNOWLEDGEMENTS}

We thank our colleagues who referred to us to Lynch patients. This study was supported by the French Canceropole Nord-Ouest and the French National Cancer Institute (INCa). We are grateful to Mario Tosi for critical review of the manuscript.

1 Lynch HT, Lynch PM, Lanspa SJ, Snyder CL, Lynch JF, Boland CR: Review of the Lynch syndrome: history, molecular genetics, screening, differential diagnosis, and medicolegal ramifications. Clin Genet 2009; 76: 1-18.

2 Vasen HF, Stormorken A, Menko FH et al: MSH2 mutation carriers are at higher risk of cancer than MLH1 mutation carriers: a study of hereditary nonpolyposis colorectal cancer families. J Clin Oncol 2001; 19: 4074-4080.

3 Ramsoekh D, Wagner A, van Leerdam ME et al: Cancer risk in MLH1, MSH2 and MSH6 mutation carriers; different risk profiles may influence clinical management. Hered Cancer Clin Pract 2009; 7: 17.

4 Alarcon F, Lasset C, Carayol J et al: Estimating cancer risk in HNPCC by the GRL method. Eur J Hum Genet 2007; 15: 831-836.

5 Zecevic M, Amos CI, Gu X et al: IGF1 gene polymorphism and risk for hereditary nonpolyposis colorectal cancer. J Natl Cancer Inst 2006; 98: 139-143.

6 Reeves SG, Rich D, Meldrum CJ et al: IGF1 is a modifier of disease risk in hereditary non-polyposis colorectal cancer. Int J Cancer 2008; 123: 1339-1443.

7 Felix R, Bodmer W, Fearnhead NS, van der Merwe L, Goldberg P, Ramesar RS: GSTM1 and GSTT1 polymorphisms as modifiers of age at diagnosis of hereditary nonpolyposis colorectal cancer (HNPCC) in a homogeneous cohort of individuals carrying a single predisposing mutation. Mutat Res 2006; 602: 175-181.

8 Pande M, Amos CI, Osterwisch DR et al: Genetic variation in genes for the xenobioticmetabolizing enzymes CYP1A1, EPHX1, GSTM1, GSTT1, and GSTP1 and susceptibility to colorectal cancer in Lynch syndrome. Cancer Epidemiol Biomarkers Prev 2008; 17: 2393-2401.

9 Wijnen JT, Brohet RM, van Eijk R et al: Chromosome 8q23.3 and 11q23.1 variants modify colorectal cancer risk in Lynch syndrome. Gastroenterology 2009; 136: 131-137.

10 Pittman AM, Naranjo S, Webb E et al: The colorectal cancer risk at 18q21 is caused by a novel variant altering SMAD7 expression. Genome Res 2009; 19: 987-993.

11 Plaschke J, Engel C, Kruger S et al: Lower incidence of colorectal cancer and later age of disease onset in 27 families with pathogenic MSH6 germline mutations compared with families with MLH1 or MSH2 mutations: the German Hereditary Nonpolyposis Colorectal Cancer Consortium. J Clin Oncol 2004; 22: 4486-4494.

12 Baglietto L, Lindor NM, Dowty JG et al: Risks of Lynch syndrome cancers for MSH6 mutation carriers. J Nat/ Cancer Inst 2010; 102: 193-201.

13 Tomlinson IP, Webb E, Carvajal-Carmona L et al: A genome-wide association study identifies colorectal cancer susceptibility loci on chromosomes 10p14 and 8q23.3. Nat Genet 2008; 40: 623-630.

14 Tenesa A, Farrington SM, Prendergast JG et al: Genome-wide association scan identifies a colorectal cancer susceptibility locus on $11 \mathrm{q} 23$ and replicates risk loci at $8 \mathrm{q} 24$ and 18q21. Nat Genet 2008; 40: 631-637.

15 Talseth-Palmer BA, Brenne IS, Ashton KA et al: Colorectal cancer susceptibility loci on chromosome 8q23.3 and 11q23.1 as modifiers for disease expression in lynch syndrome. J Med Genet 2010, e-pub ahead of print. 